

\section{Der Erweiterungsbau der WLB - Teil 8 :}

\section{Baubeginn mit Abbruch der Tiefgarage}

Am 23. März 2015 wurde der gesamte Baustellenbereich vor der WLB durch einen Bauzaun gesichert, und damit wurden die Bauarbeiten für den Erweiterungsbau endgültig eingeleitet. Dadurch sind die Grünanlage vor der Landesbibliothek sowie der Steg über die Ulrichstraße nicht mehr zugänglich. Nach Anfangsschwierigkeiten gewöhnten sich Fußgänger und Radfahrer jedoch schnell an die neue Wegeführung.

Als vorbereitende Maßnahmen für den Abbruch der Tiefgarage wurde vom 9. bis 11. April 2015 mit dem Abriss des Stegs zum Haus der Abgeordneten sowie im Anschluss daran mit den Abbrucharbeiten des Vorbaus der WLB begonnen. Zeitgleich dazu sind die umfangreichen Erd- und Styroporschichten auf dem Gelände vor der WLB abgetragen worden, um die Betondecke der Tiefgarage freizulegen.

Im Mai rückten die Abrissbagger an, die sich mit ihren tonnenschweren Greifzangen Stück für Stück in die armierten Betondecken buchstäblich hineingefressen haben. Durch die enge Verbindung zwischen der Tiefgarage und den beiden Magazingeschossen der WLB kam es - abgesehen von dem monotonen Abbruchlärm des Bohrhammers - zu nicht unerheblichen Erschütterungen und Vibrationen am Bestandsgebäude, die bei Mitarbeitern und Benutzern für einige Beunruhigung sorgten. Vonseiten der Architekten und des Amtes für Vermögen und Bau ist immer wieder darauf hingewiesen worden, dass dies leider nicht zu vermeiden sei, und man sich um die Statik des Bestandsgebäudes keine Sorgen machen müsse. Ende Juni 2015 war von der Tiefgarage bis auf die Bodenplatte nicht mehr viel zu sehen. Nach Abschluss der Abbrucharbeiten Mitte Juli konnte die Baustelle am 17. Juli 2015 der Rohbaufirma übergeben werden.

Um den Rohbau errichten zu können, werden in den nächsten Wochen für die Gründung des Erweiterungsbaus 160 Bohrpfähle gesetzt. Die damit verbundene Wasserhaltung der Baugrube hat am 15. August 2015 begonnen und kann hoffentlich bis Mitte Februar 2016 abgeschlossen werden.
Bislang jedenfalls ist der Bauzeitenplan weitgehend eingehalten worden.

Parallel dazu sind die Planungen für die Sanierung und den Umbau des Bestandsgebäudes angelaufen. Das Ministerium für Finanzen und Wirtschaft hat die Zustimmung zum Planungsbeginn und zur Erstellung der Bauunterlage erteilt. Im Zuge der künftigen Flächenbelegung im Bestandsgebäude ist der Zustand des Gebäudes sowie der technischen Anlagen im Hinblick auf den notwendigen Sanierungsbedarf untersucht worden.

Abgesehen von den für den Bibliotheksbetrieb notwendigen funktionalen Anpassungen ergaben sich folgende Schwerpunkte für die Sanierung:

- Brandschutz

- Sanitärinstallation

- Lüftungstechnik

- Sicherheitsbeleuchtung

- Aufzüge

- Schadstoffbeseitigung

Da im Estrich der Tiefmagazine Asbest festgestellt wurde, ist eine Asbestsanierung unausweichlich. Dies hat zur Folge, dass beide Tiefmagazine und das Zeitungsmagazin mit 3,5 Millionen Bänden ausgelagert werden müssen. Um den finanziellen Aufwand und die Nutzungseinschränkungen für die Bibliotheksbenutzer möglichst gering zu halten, wurde vorgeschlagen, die neue Tiefgarage temporär für die Auslagerung der Buchbestände aus den Tiefmagazinen zu nutzen. Aus Sicht der WLB ist dies die kostengünstigste Lösung, um eine Auslagerung in anzumietende Lagerflächen an der Peripherie von Stuttgart und den täglichen Fahrdienst zu vermeiden.

Diese Option wird derzeit vom Amt für Vermögen und Bau und den Architekten geprüft. Die Sanierung und der Umbau des Bestandsgebäudes wird sich unmittelbar an die Inbetriebnahme des Erweiterungsbaus Anfang 2018 anschließen, sodass die notwendigen Entscheidungen jetzt getroffen werden müssen.

Hannsjörg Kowark Martina Lüll 